\title{
LA APROPIACIÓN DE CONOCIMIENTOS A PARTIR DE LA EXPRESIÓN PLÁSTICA
}

Julieta Castro Bonilla

\author{
Recibido 5-VIII-2003 • Aceptado 12-VIII-2003
}

\begin{abstract}
Resumen: En el presente artículo se reflexiona y se expone parte de los resultados de un trabajo investigativo referido a la apropiación de conocimientos mediante la expresión plástica, actividad en la que participaron docentes y estudiantes escolares pertenecientes a la modalidad educativa denominada "Aula Abierta".

Concluida la investigación, queda manifiesto que el estudio, la indagación, la reflexión y la búsqueda sistemática de conocimientos y formas de aprender y enseñar de parte del docente, son elementos fundamentales que propician cambios cualitativos en la formación académica de éste, lo que le permite llevar al aula formas innovadoras para lograr aprendizajes significativos en sus estudiantes. El diseño de situaciones de aprendizaje desde una perspectiva constructivista, holista y lúdica, así como el aprovechamiento de contextos, permitieron el logro de aprendizajes cognitivos, afectivos y psicomotores mediante la expresión de actividades plásticas, lo que le posibilita al joven el desarrollo de una personalidad integral.
\end{abstract}

Palabras clave: Integración de Conocimientos, Constructivismo, Holismo, Recurso Lúdico, Expresión Plástica.

\section{Introducción}

Las siguientes reflexiones se refieren a la experiencia investigativa denominada "La expresión artística: un recurso heurístico para la construcción de procesos pedagógicos en el aula”, en la que participó un equipo de investigadoras y maestras que atienden estudiantes en edades comprendidas entre los ocho y dieciséis años. La experiencia permitió el logro de su objetivo: la participación sistemática de niños y jóvenes en procesos pedagógicos que mediante la reflexión, la experimentación y la aplicación de experiencias artísticas llevaron a la construcción de conocimientos, y de esta manera fortalecieron su personalidad, y en consecuencia, su propia identidad.

El artículo se origina de tres fuentes:

$1 \quad$ Desde la formación académica y profesional de la investigadora en el área de la enseñanza de las artes plásticas.

1 Como profesora universitaria impartiendo cursos relacionados con el área de las artes plásticas.

Como investigadora en una institución de educación primaria bajo la modalidad de "Aula Abierta", en la provincia de San José. 
Es importante mencionar que la mayoría de los estudiantes que participó en la investigación presenta problemas en su aprendizaje, lo que les impide la adquisición de contenidos de manera tradicional. La experiencia desarrollada fue un verdadero reto pedagógico, ya que se atendió una población con necesidades especiales que participa en un proyecto denominado "Aula Abierta”. Pese a su situación, estos alumnos deben realizar las pruebas nacionales anuales programadas por el Ministerio de Educación, sin que medie ninguna adecuación curricular en la elaboración de éstas.

\section{Métodos y procedimientos utilizados}

La investigación se desarrolló bajo un enfoque cualitativo y constructivista, fundamentada en la participación y la apropiación de aprendizajes por parte de las personas involucradas. Se utiliza una metodología participativa centrada en la realización de talleres que involucran el desarrollo de temáticas específicas del currículum escolar, mediante la elaboración de planeamientos didácticos que evidencian la presencia de sus componentes en forma coherente, flexible y contextualizados. A continuación se indican algunas de las estrategias metodológicas desarrolladas:

1 Revisión bibliográfica pertinente al tema.

1 Recopilación de artículos de interés para ser analizados conjuntamente por las investigadoras y por las docentes participantes en el proyecto.

1 Construcción de espacios académicos, en los que bajo la conducción de las investigadoras; las maestras y sus estudiantes participan en actividades que permiten la apropiación de la expresión artística como un medio para expresar emociones, sentimientos y vivencias.
1 Diseño de talleres de formación académica y planeamiento pedagógico por parte de las investigadoras y dirigidos a las docentes con el propósito de que participen en la construcción de estrategias pedagógicas a partir de la expresión artística, el constructivismo y el holismo.

1 Desarrollo de actividades pedagógicas a cargo de las docentes participantes en el proyecto, para lo que tomaron en cuenta las necesidades especiales de la población, el contexto educativo, su experiencia con este tipo de estudiantes, su creatividad y su estilo personal de trabajo.

\section{La adquisición de conocimientos mediante la expresión plástica}

Tomando en cuenta los aspectos señalados en el apartado anterior, el equipo de investigadoras planteó la forma en que abordaría el proyecto. Se trata de una propuesta investigativa novedosa que posee una fundamentación epistemológica constructivista y holista. Además, la información recopilada, analizada, observada y producida se reflexionó desde una perspectiva de género.

Con el propósito de alcanzar el objetivo propuesto, se planificaron sesiones incentivadoras con las docentes, lo que les permitió involucrarse desde su inicio en experiencias de aprendizaje integradas, en éstas se reflexionó respecto de temas como: constructivismo, holismo, género, recurso heurístico, artes plásticas y otros tópicos que incidirían directamente en el trabajo por realizar. Las experiencias de aprendizaje tuvieron un carácter de integración entre las áreas académicas propuestas mediante los programas del Ministerio de Educación Pública y la expresión plástica.

Para llevar a cabo de manera exitosa el trabajo, se desarrollaron experiencias 
con el enfoque constructivista, para lo que se aplicaron técnicas cualitativas como: entrevistas, observaciones, comentarios, sesiones de retroalimentación, ensayos y diseños de situaciones de aprendizaje, en las que se valoró el criterio de las participantes $\mathrm{y}$, de manera conjunta, se modificaron $\mathrm{y}$ reorientaron los procesos de aprendizaje.

A partir de las metas señaladas, se consideraron los siguientes indicadores de éxito:

1 Desarrollar experiencias participativas y formativas de trabajo con las maestras y sus estudiantes.

1 Gestar procesos de formación e integración de conocimientos mediante el intercambio de contenidos emanados por el Ministerio de Educación Pública y los relacionados con la expresión plástica.

1 Valorar la expresión artística como un recurso para la planificación de estrategias innovadoras.

$1 \quad$ Facilitar la integración de conocimientos mediante la expresión plástica y desde una perspectiva constructivista y holista.

Para lograr los planteamientos expuestos, fue necesario valorar la expresión plástica como un recurso heurístico (arte de inventar), que permite la apropiación, transformación y construcción del conocimiento, y que favorece la inventiva por parte de los estudiantes. Para la planificación de estrategias innovadoras se recurrió al abordaje de los "ejes transversales", desde una perspectiva didáctica y metodológica. Si se parte de que la educación escolar en su etapa obligatoria, tiene la finalidad de contribuir a desarrollar en los alumnos aquellas capacidades que se consideran necesarias para desenvolverse como ciudadanos con derechos y deberes en la sociedad en que viven, se hace urgente la formulación de procesos pedagógicos innovadores. La propuesta metodológica llevó a la práctica la aplicación del "eje transversal" denominado, en el presente trabajo, "expresión plástica". En otras palabras, este eje impregnaría al currículum escolar en su totalidad, con actividades gráficoplásticas.

Para que la propuesta se pudiera llevar a la práctica sin el mayor problema, las maestras debieron correlacionar, en primera instancia y mediante actividades debidamente planificadas, la expresión plástica con otras áreas curriculares como: música, matemática y lenguaje; de esta manera, éstas quedarían enriquecidas y redimensionadas por este tipo de actividades artísticas.

Durante el proceso, se tomaron en cuenta las diferencias individuales, así como el contexto sociocultural en que viven los alumnos, por lo que la adquisición de los contenidos tuvo un mayor nivel de significatividad y, a su vez, el área de las artes plásticas dejó de ser una asignatura más, para convertirse en un "eje transversal" que, mediante la utilización de materiales como pintura, barro, texturas $\mathrm{y}$ otros, permitió explicaciones e interpretación de contenidos curriculares de manera concreta. Es necesario mencionar que la expresión de los desarrollos: emocional, físico, perceptivo, creador y social, por parte de quien participó del proceso, forman parte de un aprendizaje más vivencial, por lo que siempre estuvieron presentes.

Otro concepto pedagógico vital en esta experiencia es el denominado "holismo", que permitió tener una visión integral del individuo como persona crítica, activa, pensante y reflexiva, para quien sus valores, su relación con el contexto inmediato y el contacto con la Naturaleza, ofrecen un mejor desarrollo de sus áreas: cognitiva, afectiva y de habilidades psicomotoras, aspecto fundamental para el proceso de la investigación.

Con una gran motivación, las investigadoras y las docentes asumieron retos, 
tareas y responsabilidades tendientes a correlacionar la expresión plástica con contenidos correspondientes a diversas áreas curriculares.

(Lucini, F. 1998, p. 43) propone que para diseñar actividades que permitan el logro de aprendizajes relevantes por parte de los estudiantes, es necesario llevar a la práctica los siguientes pasos fundamentales:

1 La definición de temas o tópicos, incluyendo el análisis de sus contenidos, así como sus implicaciones pedagógicas.

1 La determinación de la relación dinámica que pueda existir entre el eje transversal, los objetivos y los contenidos de las áreas curriculares.

$1 \quad$ Finalmente, la concreción de las pautas de actuación docente y de las estrategias didácticas o metodológicas que se seguirán para el desarrollo de los contenidos curriculares de cada una de las áreas.

Para el desarrollo de los contenidos, se consideró fundamental trabajar con los que se expresan en los programas de estudio emanados por el Ministerio de Educación Pública, por lo que fueron reconstruidos mediante aprendizajes debidamente planificados y que el alumno debió vivenciar mediante actividades de expresión plástica, tanto individuales como colectivas. La manera en que cada estudiante logró esos contenidos implicó la construcción de significados, esto es, dar sentido a lo que se aprende, de acuerdo con el planteamiento constructivista. En otras palabras, se garantizó el logro de aprendizajes significativos; gestados como logro de sus experiencias individuales y de la socialización, según lo expresa Molina Z, (1999, p.17).

Como se mencionó anteriormente, la investigación llevó a la práctica procedimientos metodológicos que involucraron, desde su inicio, la integración activa de todos los participantes durante todo el proceso. La experiencia adquirida, producto del esfuerzo investigativo, permitió vivenciar, compartir y comprender que los esfuerzos por lograr aprendizajes autónomos, creativos e integrados a contenidos curriculares específicos en los participantes de la modalidad pedagógica denominada "Aula Abierta", valen la pena, ya que para muchos de ellos, quizás, ésta será su última experiencia dentro del sistema educativo formal, por lo que se aprovechó al máximo su presencia dentro de la institución.

Cuando se toma la decisión de ser maestro o maestra, se está asumiendo la participación en una carrera que implica una vocación con compromisos de carácter personal y familiar, que involucra lo social y lo emocional. Por lo tanto, se debe tener claro que la trayectoria del docente influirá de manera definitiva en la formación académica, espiritual y emocional de sus estudiantes a través de sus vidas. Como protagonista de su función, el docente debe actualizar y dinamizar su práctica pedagógica, de manera que sus estudiantes puedan transferir sus conocimientos, actitudes y habilidades a otras experiencias y a otros contextos socioculturales; lo anterior, les ayudará a enfrentar los retos que le presenta la escuela y el ambiente en que se desenvuelven. Si se considera la educación como un arte (Pérez H., 1980), se necesitan docentes que luchen incansablemente por lograr sus sueños y permitan a sus alumnos alcanzar los de ellos; de esta manera, su labor educativa tendrá sentido, pues contribuirá a la formación de personas capaces de enfrentar sus propios retos.

\section{El artista y su expresión estética}

Para efectos de este artículo, es fundamental reflexionar respecto a algunos vocablos que permiten la ubicación en contextos específicos.

En primer término, el arte puede ser considerado desde diversas perspectivas, 
según la visión de mundo, la formación académica, el estado afectivo y emocional de quien lo interpreta. No obstante, existe un común denominador respecto al mismo, ya que se considera como el reflejo de la sociedad dentro de la cual surge. El artista concibe el arte como el resultado de su obra, como el producto de un proceso y muchas veces, como un medio de expresión capaz de resolver su vida.

Generalmente, el término "arte" supone connotaciones que se encuentran relacionadas con galerías, museos, grupos selectos o excéntricos. Para quienes hacen estas suposiciones, su producción artística se mide por los gustos personales o por patrones y cánones de belleza que varían de acuerdo con las normas de la sociedad. El arte expresado y producido por ellos se relaciona con la estética y exige la aplicación adecuada de elementos, principios y normas para que la obra sea valorada como "bella". Sin embargo, el respeto a reglas o cánones referidos a la composición y al diseño, algunas veces restringe al artista, ya que le impiden su expresión en su amplio sentido. Para el adulto, el "arte" significa crear algo nuevo a causa de alguna necesidad humana, ya sea propia o de origen social, éste debe permanecer en una constante lucha por no perder la capacidad de reflexionar, de contemplar, de manifestarse y de sentir impulsos producidos desde su interioridad a la acción y de esta manera propiciar una expresión auténtica.

\section{La expresión de sentimientos y emociones en el arte infantil}

Para quien planifica y organiza actividades artísticas que permiten la expresión de sentimientos y emociones de manera libre y espontánea, es prioritario comprender el significado que el arte posee para los niños, ya que difiere del que tiene para los adultos. Lowenfeld V. y Lambert W. (1975, p. 20) lo expresan de la siguiente manera: “...el arte infantil es una actividad dinámica y unificadora, con un rol potencialmente vital en la educación. El dibujo, la pintura o la construcción constituyen un proceso completo, en el que el niño reúne diversos elementos de su experiencia para formar un todo con un nuevo significado, en este proceso de seleccionar, interpretar y reformar esos elementos, el niño nos proporciona algo más que un dibujo, una pintura o una escultura, nos brinda una parte de sí mismo, ya que a través de este acto expresivo, podemos observar cómo piensa, cómo siente, cómo ve el mundo que le rodea, así como lo que conoce".

Para el niño, el producto que se ha materializado en una obra concreta no es lo primordial, lo más valioso es el proceso mediante el cual ha participado de manera activa, sin interrupciones, imposiciones, ni interferencias por parte de adultos.

Novaes (1973, p. 19) afirma que en la persona que crea se destacan ciertos rasgos de su temperamento, valores y actitudes emocionales, su pensamiento creativo, sus motivaciones y su percepción. En el producto creado interviene su invención y sus influencias ambientales, o sea, los condicionantes educativos, sociales y culturales; por lo que se podría interpretar que crear se refiere a la expresión o a la comunicación de lo que se tiene dentro de sí; ésta se debe dar de manera individual, dado que el esfuerzo creativo debe ser auténtico. Por lo tanto, se debe aprovechar la facultad que poseen los estudiantes para crear, imaginar y plasmar sentimientos, emociones e ideas concretas en espacios definidos y con el empleo de diversos materiales, para lo cual se deben planificar actividades artísticas que permitan la concreción de contenidos. Las siguientes preguntas permitieron que las docentes reflexionaran sobre sus planteamientos didácticos:

$1 \quad$ ¿Tendrá un niño o niña la facultad de interpretar, crear y transferir sus ideas y contenidos con la ayuda de diversos materiales?

$1 \quad$ ¿Se podrá desarrollar la creatividad en estudiantes con bajo nivel de escolaridad? 
¿Cuál es la mejor manera de desarrollar y organizar éste o aquel contenido?

¿Cuál es la mejor manera de expresar las áreas: cognitiva, afectiva y psicomotora, tomando en cuenta el eje transversal denominado artes plásticas?

Con estas preguntas reflexivas, las docentes tienen claro que el aprendizaje no se construye de manera aislada e inconexa, las experiencias personales y artísticas son fundamentales en la elaboración del conocimiento. Por lo tanto, al referirse al desarrollo de la creatividad en niños y jóvenes, se debe tener presente que se trata de procesos continuos y dinámicos que comprenden la expresión de su personalidad. La creatividad está relacionada con las características psicológicas que se presentan en cada etapa evolutiva, por lo tanto, un niño o un joven tendrá conductas creadoras relativas a sus posibilidades, a sus límites evolutivos, a sus diferencias individuales y al contexto que lo rodea. Lowenfled, V. y Lambert W. (1975, p. 39) señalan que toda expresión artística refleja los sentimientos, la capacidad intelectual, el desarrollo físico, la aptitud perceptiva, el factor creador implícito, el gusto estético y el desarrollo social del individuo; además de reflejar esos aspectos personales, también se manifiestan las transformaciones que se producen en el niño a medida que crece y se desarrolla; por ello, para comprender y valorar estos cambios, es necesario comprender el significado de los factores que componen cada desarrollo. Por lo que es recomendable que el docente y el padre de familia conozcan las características propias de cada desarrollo evolutivo con el propósito de que se comprendan y valoren de mejor manera sus experiencias artísticas. Brevemente se hará referencia a algunas de las características que conforman cada uno de los desarrollos mencionados, de acuerdo con los autores Viktor Lowenfeld y W. Lambert, (1975).
Desarrollo emocional o afectivo: se refiere a la intensidad con la cual la persona, llámese niño o joven se pueda identificar con su trabajo. Aunque este aspecto no se puede cuantificar, es necesario aclarar que el grado de autoidentificación puede variar desde la repetición de elementos de manera estereotipada, hasta un nivel alto de entrega o de compenetración con lo que hace, en donde el autor representa elementos y situaciones realmente significativas para él. La inclusión de "él o de ella", permite una participación directa en su trabajo creativo. Es importante recordar que el niño emocionalmente libre, se encuentra desinhibido en lo que respecta a su expresión creadora, se siente seguro para afrontar cualquier problema que se derive de su misma experiencia. La compenetración y el grado de intensidad con que se entregue a lo que hace, le proporciona madurez emocional y afectiva que lo favorece ante la toma de decisiones y le permite circunscribir estas experiencias a otros contextos.

Desarrollo intelectual en términos generales, este desarrollo se refleja cuando el niño que dibuja o pinta, expresa gran cantidad de detalles subjetivos. También se refiere a la forma en que dibuja el concepto de "ser humano", pues se evidencia la edad en que se encuentra. Es importante saber que a medida que éste crece, cambian los detalles y la toma de conciencia del ambiente que lo rodea. Es importante mencionar que no se pueden valorar las aptitudes intelectuales de un niño con solo observar sus dibujos. Es necesario mantener un equilibrio apropiado entre el desarrollo emocional y el intelectual, por lo que el docente debe mantener una constante motivación que le permita a sus estudiantes la adquisición del conocimiento de una manera más agradable, por lo que debe organizar actividades que resulten realmente productivas y enriquecedoras.

Desarrollo físico: se aprecia mediante la habilidad que posee el niño o la niña para su coordinación visual y motriz, así como la manera en que pueda controlar su cuerpo y pueda ejecutar con destreza su grafismo en lo que hace. La incorporación del "yo" en el dibujo, y las variaciones en su desarrollo físico pueden observarse en sus creaciones.

Desarrollo perceptivo: el estímulo y el desarrollo de los sentidos es fundamental durante el proceso creativo, por lo que se debe incentivar de manera constante y permanente, ya que de esta manera, su creación será libre y espontánea. Este desarrollo se puede apreciar, no sólo en la variedad de experiencias perceptivas que poseen sus estudiantes, sino en la manera en que pueda expresarlas. La inclusión de elementos en sus trabajos que se refieren a sensaciones como: sonidos, texturas, temperaturas, así como las experiencias kinestésicas, las formas, los colores 
y las experiencias de tipo visual y auditivo, en espacios determinados, incluyen una gran variedad de estímulos que permiten una expresión libre. Le corresponde al docente estimular el deseo de ver, de sentir, de tocar las cosas que nos rodean, ya que el estímulo de los sentidos es fundamental en el aprendizaje.

Desarrollo social: se manifiesta mediante la identificación que el niño o la niña posea de sus propias experiencias, así como la relación que establezca con los demás. A medida que crece, su expresión artística refleja un mayor conocimiento del medio social, por lo que se considera fundamental que el personal docente planifique actividades que promuevan la socialización, ya que además, este proceso le permite el desarrollo de su independencia como persona.

Desarrollo estético: Se refiere a la organización armoniosa de los pensamientos, sentimientos y emociones que realiza el niño mediante líneas, texturas, colores, en espacios determinados, esta organización de elementos se realiza de manera intuitiva. La educación desempeña un papel fundamental en el cultivo de la expresión, por lo que el docente tiene la obligación de organizar actividades que permitan la integración de otras áreas del saber: matemáticas, lenguaje, música y otras.

Desarrollo creador: se inicia cuando el niño hace los primeros trazos, ya que lo lleva a la experimentación y expresión muy personal de su grafismo, inventa sus propias formas e impone su sello personal en lo que hace.

Como se indicó anteriormente, las docentes que participaron en la investigación se dieron a la tarea de conocer las características de cada desarrollo, ya que les ayudó a comprender y a valorar mejor el trabajo elaborado por cada estudiante. En otras palabras, el responsable de planificar actividades que permitan la adquisición de contenidos mediante la expresión plástica, debe recordar que esa manifestación artística refleja no solo el grado de conocimientos alcanzados, sino su forma de ser. Se considera fundamental enfatizar que la expresión artística, específicamente, las experiencias gráfico-plásticas, favorecieron el desarrollo creativo en todas las áreas del aprendizaje. Las docentes tenían claro que la conducta creativa, las actitudes y las aptitudes que cada estudiante posee, juegan un papel determinante en el contexto escolar, ya que son ingredientes necesarios para un mejor aprendizaje. La educación en la actualidad, requiere de docentes que reconozcan que el estímulo de actividades expresivas favorece la capacidad de crear y, por lo tanto, de producir respuestas que dan sentido a lo que aprende.

Llevar a la práctica innovaciones pedagógicas que permitieran la aplicación de la "transversalidad", no fue tarea fácil para las docentes, por lo que después de mucha lectura, investigación, reflexión y análisis, se planificaron actividades metodológicas que favorecieron que los estudiantes se apropiaran del contenido y lo expresaran a su propio ritmo. Para ello, fue fundamental contar con espacios o rincones dentro y fuera de aula que permitieran observar y admirar elementos que reforzaran los conocimientos adquiridos mediante su misma construcción. Los espacios y períodos destinados a observar, descubrir, escuchar, tocar, saborear y oler, así como el refuerzo con otros recursos didácticos, “...ayudó a los estudiantes a ser más auténticos y estar más seguros de sí mismos, ingredientes fundamentales para el desarrollo de su autoestima”, expresó una de las docentes.

Los componentes que se indican a continuación hicieron posible experiencias cargadas de emociones y vivencias:

$1 \quad$ La originalidad. Se ejercitó tanto el pensamiento lógico-racional como la fluidez y libertad de ideas. Los programas emanados del Ministerio de Educación Pública dan prioridad a contenidos o temas que refuerzan este tipo de pensamiento, dejando de lado áreas 0 asignaturas que poseen un alto grado de expresividad, por lo que se impulsó el estudio de otras áreas como la expresión plástica, que promovieron la originalidad con toda libertad.

$1 \quad$ La apreciación por lo nuevo. $\mathrm{Al}$ iniciar el estudio de un tema, se llevaron a cabo de manera sistemática 
diagnósticos de los contenidos que los alumnos ya poseían, denominados también "conocimientos previos"; posteriormente, se formularon preguntas con el propósito de que los estudiantes reconocieran que nuestro conocimiento siempre estará incompleto, por lo que se insistió que debemos permanecer en una constante búsqueda. La incentivación, la exploración y el descubrimiento hacia lo novedoso, son actitudes que siempre estuvieron presentes en los participantes durante el proceso didáctico.

\section{La curiosidad y la investigación.}

Se desarrolló partiendo de la sensibilidad del alumno ante situaciones concretas, se estimuló la intuición y la aplicación de enseñanzas que invitaron a aprendizajes creativos, los que se relacionaron con otras áreas del conocimiento; también se estimuló una visión integral de la vida y de la persona.

$1 \quad$ La autodirección. Se logró permitiéndole al alumno "aprender por iniciativa propia”. Para lo que las docentes estimularon y respetaron este tipo de aprendizaje, ya que varía en cada estudiante.

1 La percepción de su propia realidad. Este apartado fue considerado vital para el estudiante, ya que las docentes en todo momento permitieron que cada quien expresara su propia realidad, su propio mundo, su yo interno, el que fuera construido desde su nacimiento y a través de su vida se fue transformando y modificando, le corresponde al maestro aceptar y respetar la percepción de la realidad que cada uno posee.

El aprendizaje es un proceso complejo, en el que interviene no solamente la capacidad intelectual, sino otros factores como los sociales, emocionales, perceptivos, físicos y psicológicos. Para que el niño pueda ser capaz de expresar con toda libertad sus sentimientos y emociones, debe haber participado en procesos de aprendizaje realmente significativos para él. Para ello, las docentes organizaron actividades en las que se invitaba al descubrimiento, a la exploración y a la investigación de temas relacionados con su estudio.

\section{Condiciones que propician el desarrollo de la capacidad expresiva}

A continuación se mencionan las actitudes de tipo afectivo y condiciones académicas que debe poseer quien tenga la responsabilidad de lograr que sus estudiantes se expresen libremente.

Acerete D. (1974) sitúa la expresión artística como sinónimo de acción, tanto en la vida social como individual. Desde esta perspectiva, se debe tener claro que el desarrollo de la sensibilidad por parte del docente para comprender el significado de las formas, la práctica de los medios de expresión: dibujo, pintura, modelado y otros, es fundamental para el desarrollo de sus facultades creadoras.

El desarrollo de los campos sensoriales y emocionales mediante estrategias novedosas fue fundamental para la relación que se debió establecer entre el "eje transversal", denominado en este trabajo "artes plásticas”, y otras áreas del currículum 
escolar, ya que permitió a los estudiantes la transmisión de sus formas de pensar de manera espontánea y libre. Lo anterior implicó un "proceso", en el que después de participar en la construcción de conocimientos, los estudiantes seleccionaron, interpretaron y transformaron a su manera y con un significado muy personal lo aprendido. Posterior a este proceso, el estudiante se encontraba en capacidad de expresar lo que había comprendido, posterior al estudio de un tema, ya que se encontraba en capacidad de transferir a otras situaciones o contextos lo aprendido, y por lo tanto, le permitía al docente valorar lo adquirido por sus estudiantes.

El propósito de la aplicación de la "transversalidad", no es formar "niños-artistas"; sin embargo, el docente espera que el proceso artístico sirva como punto de partida para que puedan llevarse a cabo experiencias de arte mediante la construcción de sus propias formas de comunicación y de expresión.

Las experiencias de arte producidas en el aula son fuentes inagotables de motivación, ya que toda experiencia artística ayuda al estudiante a encauzar su imaginación. Aunque en los procesos de enseñanza y aprendizaje, todos sus participantes tienen el mismo nivel de importancia, el docente es considerado como el gestor de procesos pedagógicos que inviten a verdaderos aprendizajes por parte de sus estudiantes; por lo que es conveniente que éste posea excelentes condiciones emocionales, personales y académicas que le permitan crear atmósferas que conduzca a la inventiva, a la imaginación, a la exploración y a la producción.

Algunas de las cualidades personales que debe poseer quien organiza actividades lúdicas se citan a continuación:

En primer lugar, debe ser una persona cordial, respetuosa, amable, creativa y flexible. Ha quedado en evidencia que la calidad del trabajo producido por los alumnos depende en gran parte del comportamiento $\mathrm{y}$ actitud positiva del docente. Una actitud de amistad, de cordialidad y de interés por los estudiantes, favorece la expresión libre y espontánea, que debe ser traducida en "un producto artístico de calidad", que realmente refleje lo que es como persona. Para que el proceso sea realmente significativo, el ambiente de aula debe estar marcado por una atmósfera que conduzca a la creatividad, por lo que es fundamental que las experiencias pedagógicas culminen con "productos concretos".

$\mathrm{Al}$ leer sobre las actividades metodológicas de expresión plástica que permitan una manifestación libre y espontánea, nos encontramos con una variedad de posturas y métodos que reflejan una enorme gama de consideraciones valorativas de acuerdo con el contexto. Sin embargo, resulta fundamental preguntarse si la aplicación de la "transversalidad", realmente permite la adquisición de contenidos curriculares, así como el desarrollo de la persona de manera integral. Aunque la respuesta a esta pregunta resulte difícil de determinar, pues no se pueden ofrecer "recetas", las educadoras que participaron lo hicieron de manera responsable, ya que se involucraron desde un inicio en la investigación, estudio e implicaciones que ésta conlleva, su participación de los talleres de sensibilización permitieron un mejor desarrollo académico, personal y emocional; ingredientes fundamentales para llevar a la práctica métodos y técnicas que resultaron idóneas para la programación de actividades que implicaron enseñanzas novedosas, tomando en cuenta diversas formas de aprender.

\section{Conclusión}

Para concluir, deseo reflexionar en torno a la calidad de los procesos pedagógicos que las docentes organizaron, ya que permitieron el logro de aprendizajes significativos de parte de sus estudiantes escolares. A continuación se indican algunos de ellos: 
$1 \quad$ Las actividades planificadas por las docentes participantes evidenciaron cambios de actitud significativos de sus estudiantes, ya que el replanteamiento novedoso llevado a cabo en sus prácticas pedagógicas, permitieron una mayor participación de parte sus estudiantes, tanto en actividades curriculares dentro como fuera del aula.

En la elaboración de planteamientos metodológicos se evidencian los siguientes fundamentos: constructivismo, holismo y la expresión plástica, como eje transversal. Estos permitieron el desarrollo de actividades lúdicas y de aprendizajes significativos mediante la integración de contenidos curriculares. Lo expuesto se manifiesta en el siguiente comentario que hace una de las docentes: "En primera instancia, el trabajo en el que participé me permitió hacer un cambio de paradigma en mi vida, los replanteamientos que sustentan ahora mi práctica pedagógica, me permiten hacer ajustes para modificar mi trabajo de aula de manera positiva”.

La planificación de actividades didácticas realizadas por las maestras, bajo la asesoría de las investigadoras, se organizaron de una manera creativa y lúdica ya que la "expresión plástica" permitió el logro de contenidos de una manera más vivencial. Una docente indica "mi participación en esa investigación me permitió conocer el concepto de holismo (desconocido para mí) y de esta manera poder entrelazar la creatividad, expresión artística y el constructivismo con el currículum escolar. Este nuevo enfoque me ha permitido tener una visión de trabajo de aula diferente".
Las docentes mencionan los siguientes logros obtenidos por su participación en el proyecto:

$1 \quad$ “...un mayor conocimiento de los siguientes temas: constructivismo, holismo, creatividad y la incidencia de éstos en los procesos de enseñanza y aprendizaje".

"Concluida mi participación, creo que puedo hacer una mejor distribución del tiempo y del espacio, además, la aplicación de ejes transversales en el planeamiento de actividades, permiten una participación de parte de los niños mucho más placentera, sobre todo tomando en cuenta el tipo de población estudiantil con que trabajo".

1 "Los cambios cualitativos los puedo evidenciar de manera positiva, ya que me intereso más en mí misma como persona. Ahora me intereso realmente por las artes plásticas ya que me permiten conocer lo que han aprendido mis estudiantes, como educadora me siento más responsable”.

1 "Los escolares participantes valoran las experiencias desarrolladas en el aula como interesantes, diferentes, nuevas, agradables y activas, según lo apuntaron en el instrumento de evaluación que completaron al final de la investigación”.

El equipo de investigadoras considera que, "Como parte de su cotidianidad, las docentes comprendieron la necesidad de reflexionar de manera sistemática acerca de su práctica pedagógica, ya que incursionaron en nuevas formas de enseñar y de lograr mejores resultados en el aprendizaje de sus estudiantes". Asimismo, los talleres de sensibilización permitieron a las maestras un mayor conocimiento de ellas 
mismas, lo que hizo posible un mejor trabajo de aula. Finalmente, se comprendió que el contexto de aula y el escolar deben estar encaminados a facilitar los procesos de construcción y apropiación del conocimiento, así como el desarrollo de habilidades, actitudes y aptitudes, lo que permite el desarrollo armonioso e integral de estudiantes, especialmente a los que pertenecen a poblaciones vulnerables como la que participara en la investigación.

\section{Referencias bibliográficas}

Álvarez, María Nieves y otros. Valores y temas transversales en el currículum. Barcelona España. Editorial Grao. 2000.

Beuchat, Cecilia R. y otros. Desarrollo de la expresión integrada: club cli-clopips: Santiago de Chile: Editorial Andrés Bello. 1994.

Cherry, Clare. El arte en el niño en edad preescolar. Barcelona España: Ediciones CEAC, S.A. 1978.

Gardner, Howard. Educación Artística y Desarrollo Humano. Barcelona Es- paña: Editorial Paidós Educador. 1999.

Lowenfeld, Víctor y Lambert, W. Desarrollo de la Capacidad Creadora. Buenos Aires: Editorial Kapeluz. 1975.

Lucini, Fernado. Temas transversales y áreas curriculares. Madrid, España. Ediciones Anaya. 1999.

Molina, Zaida. "Planificación, Diseño y Desarrollo Curricular". Revista Umbral. Colegio de Licenciados y Profesores en Letras, Filosofía, Ciencias y Artes. San José Costa Rica.

Novaes, María Helena. Psicología de la Aptitud Creadora. Buenos Aires. Editorial Kapeluz. 1973.

León, Ana Teresa. El Maestro y los Niños, la humanización del aula. San José: Editorial de la Universidad de Costa Rica. 1998.

Klingler, Cynthia y otros. Psicología Cognitiva. Estrategias en la práctica docente. México. Editorial Mc. Graw Hill. 1999.

Julieta Castro Bonilla Investigadora en el Instituto de Investigación para el Mejoramiento de la Educación Costarricense (IIMEC) 